\title{
A computer tool for automatic braking distance calculation
}

\author{
C. Sicre ${ }^{1}$, A. P. Cucala ${ }^{1}$, A. Fernández ${ }^{1} \&$ L. Cano ${ }^{2}$ \\ ${ }^{1}$ Instituto de Investigación Tecnológica, Escuela Técnica Superior de \\ Ingeniería, Universidad Pontificia Comillas, Madrid, Spain \\ ${ }^{2}$ Spanish Railway Infrastructure Administrator (ADIF), Spain
}

\begin{abstract}
This paper presents a new algorithm and a procedure for the efficient and accurate calculation of braking distances satisfying current safety Standards. One of these Standards is applied in ADIF (Spanish Railway Infrastructure Administrator), for instance, in the design of signalling systems, to locate signals and balises. Following the Standard, braking distance tables depend on the average gradient along that distance and the speed reduction. In practice, an initial slope is calculated over a forecasted braking distance along the railway track, so that if the corresponding braking distance given by the Standard highly differs from the initial one, it will be necessary to recalculate the average gradient and apply the Standard again. The manual application of this process may end up overestimating braking distances unnecessarily (as the Standard itself includes safety coefficients), falling into additional signalling costs and reducing the line capacity. This paper presents an iterative calculation procedure to adjust as much as possible the distance required by the Standard, so that the average gradient introduced into the Standard corresponds precisely with the braking distance where the average gradient has been computed. Furthermore, the algorithm improves the accuracy of the average gradient calculation, considering the slope transition curves instead of sharp slope vertices. The proposed algorithm has been implemented in a computer tool for its use in railway lines operated by ADIF, according to the braking distance current Standard (N.T.C.020.95). This tool incorporates a railways net model that allows the user to introduce a final braking point, a speed reduction, and provides automatically the kilometric line point where the brake should start. In addition, this tool provides a systematic procedure to obtain braking distances in the different technical departments of ADIF. This paper compares, in real cases, the non-assisted previous procedure with the application of the proposed one, showing the refined braking distances.
\end{abstract}

Keywords: braking distance, N.T.C.020.95, KV, average gradient, signalization. 


\section{Introduction}

The analysis of a train braking process is necessary for a wide range of applications, as the calculation of travel times and consumptions [1], the calculation of the minimum interval between trains [2], the design of energy efficient driving [3], etc. Researchers and designers may apply different techniques that fit each problem, based on simulation or optimisation. However, for specific applications with safety implications, the calculation method must be ruled and Standardised by the Railway authority, for instance for signalling design to locate signals or balises.

Some countries like Australia are in favour of standardising the calculation method. In [4], the authors present a braking distance calculation method that depends on the line gradient, the brake delay time, the mass, deceleration rate and the speed of the train. The line gradient is considered as a list of slopes changing abruptly on the vertices, but they are not grouped into a single average gradient, that is, distance is calculated on each section of constant slope.

In other countries like UK or Spain, the signal designers rely on published Standards containing previously calculated braking distances. In UK this information is in the Braking Distance Speed Graphs, published by Railtrack in 1996 [5]. In Spain, the current Standard for braking distances is N.T.C.020.95 [6], published by RENFE and applicable since 1995. This Standard contains a set of tables where three inputs are required to search the corresponding braking distance for a speed reduction: the initial and final speed of the train and the average gradient of the railway track during the braking process. The distances given by the Standard are considered safe, and they include coast delay time (delay to cut traction), brake delay time (time to apply brakes) and other safety factors, so they can be applied directly to locate signals or balises.

The process of calculating braking distances using the Standard is iterative, due to the fact that one of the three inputs, the average gradient of the railway track during the braking process cannot be calculated until the braking distance is known, so the iterative process must start forecasting an initial average gradient. When the process is applied manually, it usually ends up overestimating unnecessarily braking distances, which carries out the following consequences:

- From the safety point of view, it is not necessary, as the Standard already includes brake delay time, coast delay time, and other safety factors that guarantee the brake.

- The cost of the infrastructure increases, as the longer the braking distance, the longer the communication wire must be.

- The capacity of the line is reduced. Capacity depends on distance between signals, so when the distance increases, so does the minimum interval between two trains, and capacity decreases [7].

Furthermore, manual calculation usually simplifies the calculation of the average gradient, considering only a list of changes of slope, joined by sharp vertices, what ends up with a low accurate value.

The purpose of the algorithm presented in this paper is the automatic and systematic calculation of braking distances in a more efficient and accurate way 
satisfying the current Standard. This is achieved thanks to two main contributions: the iterative calculation of the braking distance until the track section where the average slope is calculated coincides with the distance provided by the Standard, and the consideration of the transition curves between two different slopes. The implementation of this algorithm and a railway net model in a computer tool allows the user to introduce a final braking point, and to obtain the kilometric rail point where the braking process must start.

\section{Architecture of the algorithm}

The algorithm is based on a basic architecture shown on figure 1. It consists of three main modules related among them: the braking distance module, the average gradient module, and finally, the kilometric translator module.

\subsection{Braking distance module}

The braking distance module is a Database containing all the tables of braking distances shown in Standard N.T.C.020.95, for complete stop and for reduction of speed. The input data for this module are the initial and final speed of the train and the average gradient during the braking distance, and the output is the corresponding braking distance found in the Standard. Both speed reduction and average gradient are tabulated discretely. For example, the Standard has a braking distance table for reductions from $160 / 150 \mathrm{~km} / \mathrm{h}$. Next table is for reductions from $140 / 130 \mathrm{~km} / \mathrm{h}$, so if a train travels at $143 \mathrm{~km} / \mathrm{h}$, the table to use is the corresponding to $160 / 150 \mathrm{~km} / \mathrm{h}$.

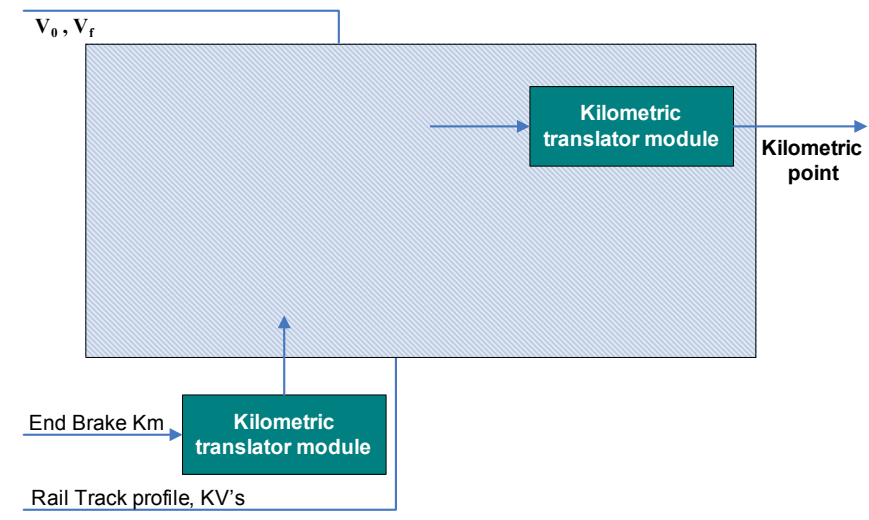

Figure 1: $\quad$ Architecture of the braking distance algorithm.

\subsection{Average gradient module}

The average gradient module performs the accurate computation of the average gradient before the speed reduction point. The input data for this module are the braking distance given as output by the braking distance module, as well as the 
elevation profile of the railway track, and the kilometric point where the brake must finish. As a result, this module calculates the average gradient of the railway track along the input distance immediately before the end braking point.

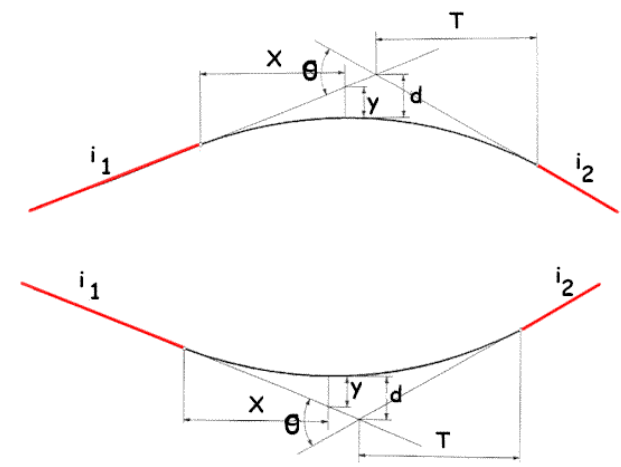

Figure 2: $\quad$ Transition Curves between different slopes.

As previously described, this module contains one of the main contributions to the increase of the accuracy in the calculation process: transition curves between two different slopes are modelled, instead of sharp vertices. In general, the function used to define the curve transition can be approximated as a parabolic curve modelled by the equation $x^{2} /(2 \cdot K v)$. Parameter $K v$ can be approximated as $L / \theta$, being $L$ the curve length, and $\theta$ the absolute value of the algebraic difference between the slopes in the extremes, $\left(i_{1}, i_{2}\right.$ in Figure 2$)$.

If the information of the $K v$ 's is not available, the algorithm will consider transitions as vertices, but the application of this curves this can sometimes shorten or enlarge significantly the distances, as it will be shown later in section 3 .

\subsection{Kilometric translator module}

The kilometric translator module applies the equivalence table between the kilometric points of the railway track and the kilometric points of the train service for the considered route. The kilometric point of the railway track corresponds to the physical kilometric signalization of a specific point on the rail, and the kilometric point of the train service is the distance of this point from the origin of the train service. The input data corresponding to the reduction point where the brake process must finish is entered by the user as the physical location on the track, thus it must be translated by this module into a kilometric point of the train service and this result is given to the average gradient module (see Figure 1). The symmetrical translation must be performed when the iterative process is finished and the braking distance has been computed, to locate the starting braking point on the railway track, that is, to locate the position of the balise or the signal on the railway track, which is the final output of the tool. 
The three reasons why the kilometric point of the railway track and the kilometric point of the train service may differ are:

- Different distance reference. When the origin to measure distances in a line is not the departure point of a train service it is necessary a kilometric translation. For instance, the kilometric origin in line Madrid-Córdoba is located in Madrid, and a train circulating from Córdoba to Madrid would have its own distance reference in Córdoba.

- The distance travelled by a train is not the difference between the corresponding track kilometric points. Typically the distance travelled along a double track line is not exactly the same for both circulation senses. However, the kilometric signalization is unique for both tracks of the line, thus differences will be found when comparing travelled distances with track kilometric points. For instance, a train travelling to Madrid through line Madrid-Sevilla shows the equivalence Table 1. This table shows that $118,497-107,650 \neq|36,018-46,843|$, so a conversion between the kilometric signalization on the track and the real distance travelled by the train is needed.

Table 1: $\quad$ Railway track and train service kilometric equivalence.

\begin{tabular}{|c|c|c|}
\hline & $\begin{array}{c}\text { Rail Track } \\
\text { km }\end{array}$ & $\begin{array}{c}\text { Train Service } \\
\text { km }\end{array}$ \\
\hline & 118,497 & 36,018 \\
\hline & 107,650 & 46,843 \\
\hline & 5,400 & 149,093 \\
\hline Madrid & 0,000 & 154,482 \\
\hline
\end{tabular}

- $\quad$ Concatenation of rail sections. Some routes are composed of consecutive rail sections each with a different kilometric origin point. In this case, the kilometric signalization on the railway track is discontinuous, but the kilometric points of the train service are always increasing with the train travelled distance. For example, in the Madrid-Málaga route, a train travels first along the line Madrid-Sevilla up to the deviation in Córdoba, and then the route continues along the line Córdoba-Málaga, with a different kilometric origin (see table 2).

Table 2: $\quad$ Railway track and train service kilometric equivalence.

\begin{tabular}{|c|c|c|c|}
\hline & $\begin{array}{c}\text { Rail Track } \\
\text { km }\end{array}$ & $\begin{array}{c}\text { Train Service } \\
\mathrm{km}\end{array}$ & \\
\hline $\begin{array}{l}\text { Line 1, Madrid - Sevilla } \\
\text { until Córdoba }\end{array}$ & $\begin{array}{c}0,000 \\
358,090\end{array}$ & $\begin{array}{c}0,000 \\
358,090\end{array}$ & Distance from the origin to \\
\hline Line 2, Córdoba - Málaga & $\begin{array}{c}0,000 \\
154,516\end{array}$ & $\begin{array}{l}358,091 \\
512,607\end{array}$ & the end : Madrid - Málaga \\
\hline
\end{tabular}

\subsection{Computation process}

The braking distance module forms with the average gradient module a circular structure. An iterative process is performed before showing the definitive 
braking distance to achieve a desired grade of accuracy in the final result, as neither the average gradient during the braking distance, nor the braking distance itself are initially known. So, the first assumption that the application uses to start the iterative process is that the average gradient is cero, and the stop condition that ends with the iterative process is when the difference between the braking distance given in the $n-1$ step and the one given in the step $n$ is small enough ( $1 \mathrm{~m}$ for the examples on section 3$)$.

With the initial and the final speed, and supposing initially a null average gradient, the braking distance module searches into the Standard N.T.C.020.95 tables and gives an initial braking distance. Then, that braking distance is introduced in the average gradient module, starting the iterative process. The application calculates the average gradient during that distance searched in the previous step, which may differ from the one that was supposed in the first step. In the second iteration, when again, the initial and final speed of the train and this new calculated average gradient are used altogether to search into the N.T.C.020.95 tables and obtain a new braking distance. The iteration process is finished when the distance required by the Standard has been calculated with the desired accuracy.

\subsection{Implementation}

The braking distance algorithm has been implemented in a computer tool that consists of two different parts; the library that contains the algorithm itself, and the interface files from where the library is called. The library is programmed in VBA above Microsoft Excel, and can be installed as a complement in it, thus allowing Microsoft Excel interface files to call it.

The interface file contains the list of braking points and speed reductions to calculate the corresponding braking distances, the line information required to apply the algorithm, as well as the output provided for each reduction: braking distances, location on the rail net and average gradients. The line information can be loaded from external .xls files thus the information can be organised to have each line in a different file. These files contain a complete list of slopes, KV's and the kilometric translation table.

\subsection{Table generation}

In addition another module was modelled and implemented to generate automatically the tables contained in the Standard. For that purpose, additional inputs parameters were considered:

- $\quad$ The brake force percentage and $\varphi$ (adherence coefficient), dependent on the initial speed of the train, and increasing with the initial speed.

- A reduction coefficient for average gradients between -21 and -25 to avoid braking distances being excessively long, always under Standard requirements.

- $\quad$ Brake and coast delay times, both dependent on the initial speed. 
Once this calculation module was validated checking that the distances calculated and the ones contained in the Standard coincide, the same algorithm was executed to generate more braking distance tables with lower discrete steps of speed and slope, thus again contributing not to overestimate braking distances.

For example, in a reduction from 90 to $50 \mathrm{~km} / \mathrm{h}$ with an average gradient of $7 \%$, the Standard would provide a braking distance of $770 \mathrm{~m}$ (the applicable table is for reductions from $100 / 90 \mathrm{~km} / \mathrm{h}$, with an average gradient of $-10 \%$ ). Nevertheless, with the table generator a new table for reductions from $90 \mathrm{~km} / \mathrm{h}$, with an average gradient of $-7 \%$ can be calculated and the obtained distance is $703 \mathrm{~m}, 67 \mathrm{~m}$ less than the previous one.

Furthermore, new tables were generated corresponding to initial speed over $160 \mathrm{~km} / \mathrm{h}$, providing valuable information related to braking distances that the algorithm (the actual Standard is based on it) would provide for higher speeds and it could be used for a possible extension of the Standard. Finally, the smallest braking distance found in the Standard N.T.C. 020.95 is $500 \mathrm{~m}$ that is set as the minimum value provided, however it can be easily checked that the distance calculated by the table generator algorithm is less than $500 \mathrm{~m}$.

\section{Examples}

This section will present the main advantages of the algorithm through the following three examples:

\section{Example 1}

The first example compares the distance calculated manually in Adif for a speed reduction in a railway line in Spain to locate a real balise, and the distance that the algorithm obtains following the previously described process.

In the manual process the engineer first estimates the distance required for the speed reduction. Then, consecutive sections of constant slope are added up to a value that is approximately the forecasted distance and the average gradient of that set of sections is calculated without considering slope transitions. Given that average gradient, the initial and the final speed, the Standard provides a value of braking distance. If that value is greater than the previous set of sections, the next section with a constant slope is added, the average gradient is recalculated and a new braking distance is obtained and compared with the previous set of sections. This process finishes when the output of the Standard is less than the length of the set of sections. At the end, to correct possible errors inherent to this method, a safety additional distance is added.

The study case has the following slope sections immediately before the end braking point:

$(6.988 \cdot 721+16.986 \cdot 692+10.315 \cdot 390) /(721+692+390)=7.92 \%$. Given the input data $\mathrm{V}_{0}=200 \mathrm{~km} / \mathrm{h}, \mathrm{V}_{\mathrm{f}}=0 \mathrm{~km} / \mathrm{h}$ and an average gradient of $8 \%$, the distance obtained from the Standard was incremented by an additional safety distance, and the balise was finally located $2.275 \mathrm{~m}$ before the end braking point. 


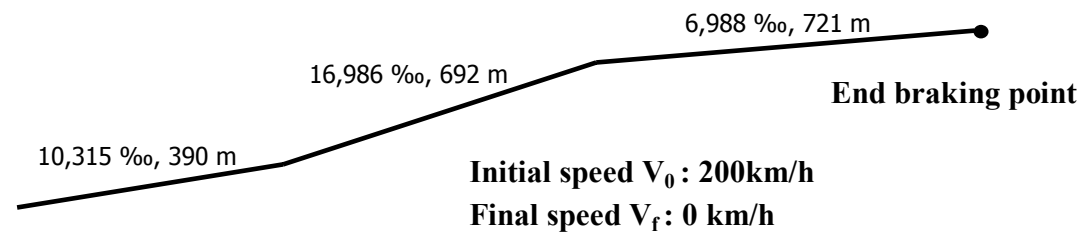

Figure 3: The manual method would give an average gradient of $7,92 \%$ :

The manual process overestimates distances because it adds whole discrete sections of constant slope. On the other hand, the application of the automatic and iterative proposed algorithm allows the accurate calculation of the distance required by Standard N.T.C. 020.95 (in this example the accuracy is set to $1 \mathrm{~m}$ ). In addition, the exact average gradient is calculated $(11 \%)$ considering slope transition curves. The resulting braking distance is $2.111 \mathrm{~m}, 164 \mathrm{~m}$ less than the previous one. It has to be taken into account that such improvement of the process calculation is possible thanks to computer calculation and automation power.

\section{Example 2}

In this example, the isolate influence of transition curves between different slopes in the braking process calculation is shown in the line Madrid-Toledo, with typical speed reductions along the drive. The following table shows the differences between considering transition curves or not.

Table 3: Comparison between considering and not considering slope transitions.

\begin{tabular}{|c|c|c|c|c|c|c|c|c|c|}
\hline Initial & Final & Braking & \multicolumn{2}{|c|}{ Without slope transitions } & \multicolumn{2}{|c|}{ With slope transitions } & \\
\cline { 6 - 9 } Speed & Speed & End & Start & Distance & Average & Start & Distance & Average & Save \\
\hline $\mathrm{km} / \mathrm{h}$ & $\mathrm{km} / \mathrm{h}$ & Track $\mathrm{km}$ & Track km & $\mathrm{km}$ & gradient & Track km & $\mathrm{km}$ & gradient & $(\mathrm{m})$ \\
\hline 160 & 140 & 10,30 & 9,689 & 0,611 & 5 & 9,693 & 0,607 & 6 & 4,000 \\
160 & 110 & 32,90 & 31,807 & 1,093 & -7 & 31,807 & 1,093 & -7 & 0,000 \\
160 & 100 & 56,10 & 54,894 & 1,206 & -7 & 54,956 & 1,144 & -3 & 62,000 \\
100 & 60 & 50,00 & 49,228 & 0,772 & -5 & 49,228 & 0,772 & -5 & 0,000 \\
\hline
\end{tabular}

In case number 1 the accurate calculation of the braking distance allows to reduce the required distance in $4 \mathrm{~m}$, and in case 3 the distance is reduced in $62 \mathrm{~m}$.

\section{Example 3}

This last example shows that improved distances can be calculated if the braking distance tables in the Standard are complemented with new tables to reduce speed and slope steps. In table 4 braking distances are displayed for initial speeds of 80,85 and $90 \mathrm{~km} / \mathrm{h}$ and final speed of $0 \mathrm{~km} / \mathrm{h}$.

The initial speed step in the Standard N.T.C.020.95 is $10 \mathrm{~km} / \mathrm{h}$, in consequence the braking distance for a train with an initial speed of $85 \mathrm{~km} / \mathrm{h}$ would be the corresponding to the initial speed of $90 \mathrm{~km} / \mathrm{h}$ in the Standard $(1.541 \mathrm{~m})$, which is overestimated. The proposed algorithm (the same algorithm that generate the tables in the Standard) can generate a new braking distance 
Table 4: $\quad$ Example 3. Braking distances with smaller speed steps.

\begin{tabular}{|c|c|c|c|c|c|}
\hline $\begin{array}{c}\text { Initial } \\
\text { Speed }\end{array}$ & $\begin{array}{c}\text { Final } \\
\text { Speed }\end{array}$ & $\begin{array}{c}\text { Braking } \\
\text { End }\end{array}$ & $\begin{array}{c}\text { Start of } \\
\text { braking }\end{array}$ & $\begin{array}{c}\text { Braking } \\
\text { distance }\end{array}$ & $\begin{array}{c}\text { Average } \\
\text { gradient }\end{array}$ \\
\hline $\mathrm{km} / \mathrm{h}$ & $\mathrm{km} / \mathrm{h}$ & Track $\mathrm{km}$ & Track $\mathrm{km}$ & $\mathrm{km}$ & $\% \circ$ \\
\hline 80 & 0 & 5,000 & 3,668 & 1,332 & -20 \\
85 & 0 & 5,000 & 3,587 & 1,433 & -20 \\
90 & 0 & 5,000 & 3,459 & 1,541 & -20 \\
\hline
\end{tabular}

table for an initial speed of $85 \mathrm{~km} / \mathrm{h}$, providing as a result a braking distance of $1.433 \mathrm{~m}, 108 \mathrm{~m}$ less $(7 \%)$ than the previous distance.

\section{Conclusions}

This paper has presented the algorithm implemented on a software tool for the Spanish Railway Infrastructure Administration Adif to perform automatic and systematic and accurate braking distance calculation according the current Standard N.T.C. 020.95 .

This Standard is used to locate signals and balises and contains a set of previously calculated braking distances, containing safety factors that depend on the speed reduction and the average gradient along the braking distance. The manual procedure typically applied in Adif to obtain the braking distance required by the Standard, tends to overestimate these distances, falling into additional signalling costs and reducing the line capacity.

The proposed algorithm improves the calculation of braking distances due to its iterative execution to obtain the distance required by the Standard with a predefined accuracy ( $1 \mathrm{~m}$ for instance), as well as the consideration of the transition curves between two different slopes.

This paper has compared real examples of braking distances obtained by the manual procedure and by the proposed algorithm, where reductions up to $11 \%$ of the braking distance could be achieved. The systematic application of this algorithm on future Adif lines will improve capacity and reduce signalling costs, observing the safety criteria according to the current Standard.

\section{References}

[1] P. Lukaszewicz, "Energy Consumption and Running Time for Trains, " in KTH, Department of Vehicle Engineering: Royal Institute of Technology, Stockholm, 2001, pp. 153.

[2] F. de Cuadra, A. Fernández, and J. C. Granados, "Train simulation and headway calculations: an approach based on parametrised, " in Computers in Railways IV, Vol 1 Railway Design and Management: WIT Press, 1994.

[3] F. de Cuadra, A. Fernandez, J. de Juan, and M. A. Herrero, "Energy-saving automatic optimisation of train speed commands using direct search techniques," in Computers in Railways V - Vol.1 Railway Systems and Management, 1996. 
[4] Barney, D., Haley, D. and Nikandros, G. (2001). “Calculating Train Braking Distance". In Proc. Sixth Australian Workshop on Industrial Experience with Safety Critical Systems and Software, Brisbane, Australia. Conferences in Research and Practice in Information Technology, 3. Lindsay, P., Ed. ACS. 23-30.

[5] Standard GK/RT0038, "Speed Restrictions", Railway Group Standard, RAILTRACK PLC (Safety \& Standards Directorate), May 1996.

[6] "Distancias de Frenado y Señales N.T.C. 020.95 Norma Técnica de Circulación," RENFE, Circulación Dirección de Inspección y Seguridad, Gerencia de Condiciones de Circulación de Material, 1995.

[7] J. Pachl, Railway Operation and Control. ed. Rail Publishing, Germany, 2002, pp 147-156. 